\title{
Effect of HPBW on channel capacity in near-field MIMO system
}

\author{
Dalin Zhang ${ }^{\text {a) }}$, Toshikazu Hori, and Mitoshi Fujimoto \\ Graduate School of Engineering, University of Fukui \\ 3-9-1 Bunkyo, Fukui 910-8507, Japan \\ a) zhang@wireless.fuis.u-fukui.ac.jp
}

\begin{abstract}
Considering the very short distance between transmitter and receiver in the near-field MIMO system, the impact of the radiation pattern of each antenna element is not neglectable. In this paper, the dual-dipole arrays are utilized to investigate the effect of HPBW (halfpower beamwidth) on channel capacity. The HPBW can be changed by varying the internal distance between two dipoles in one element. With a proper HPBW of the antenna element, the channel capacity of a dual-dipole array is improved obviously from a conventional dipole array. And the optimum HPBW is found to be approximately $50^{\circ}$.
\end{abstract}

Keywords: MIMO, near-field, dual-dipole, HPBW, channel capacity Classification: Antennas and Propagation

\section{References}

[1] Y. Karasawa, "Innovative antenna and propagation studies for MIMO systems," IEICE Trans. Commun., vol. E90-B, no. 9, pp. 2194-2202, Sept. 2007.

[2] N. Honma, K. Nishimori, T. Seki, and M. Mizoguchi, "Short range MIMO communication," Proc. EuCAP2009, Berlin, Germany, pp. 1763-1767, March 2009.

[3] K. Nishimori, N. Honma, T. Seki, and K. Hiraga, "On the transmission method for short-range MIMO communication," IEEE Trans. Veh. Technol., vol. 60, no. 3, March 2011.

[4] J. Jiang and M. A. Ingram, "Spherical-wave model for short-range MIMO," IEEE Trans. Commun., vol. 53, no. 9, pp. 1534-1541, Sept. 2005.

[5] F. Bohagen, P. Orten, and G. Oien, "Optimal design of uniform rectangular antenna arrays for strong line-of-sight MIMO channels," EURASIP J. Wirel. Commun. Netw., vol. 2007, Artical ID 45084, 10 page.

[6] G. J. Foschini and M. J. Gans, "On limits of wireless communications in a fading environment when using multiple antennas," Wireless Personal Communications, vol. 6, no. 3, pp. 311-335, March 1998.

\section{Introduction}

MIMO (Multiple-Input-Multiple-Output) [1], which has a wider bandwidth, 
multi-value modulation system, and spatial multiplexing scheme, is employed in the high-speed NFC (near-field communication) systems [2]. Contrary to conventional MIMO systems, near-field MIMO communication systems transfer data in a very short range [3], and the LOS (line-of-sight) paths are the major components $[4,5]$. Considering the short distance, the LOS components from each of the Tx elements arrive at the Rx array with a spherical wavefront [5]. Therefore the beamwidth of the antenna element radiation pattern affects not only the receiving gain but also the spatial correlation characteristics. Consequently, the impact of the radiation pattern of each antenna element can't be neglected. Usually, the conventional dipole antennas are used to investigate the MIMO channel capacity. However, the conventional dipoles are omni-directional in the horizontal plane. Therefore, in this paper, a directional element named dual-dipole element is proposed to clarify the impact of the variation of the HPBW (half-power beamwidth) in the radiation pattern.

This paper firstly introduces the dual-dipole element in the near-field MIMO. The proposed directional element consists of two dipoles in parallel, and it has a bi-directional radiation pattern in the horizontal plane. We can adjust the HPBW by changing the internal distance between two dipoles in one element. Next, it is shown that employing a proper HPBW can obtain the impressive improvement on the channel capacity. And the effect of the HPBW on channel capacity is explained in detail and analysed by eigenvalues. Finally, the system is discussed in different situations to clarify the generality of the optimum HPBW.

\section{Near-field MIMO with dual-dipole array}

\subsection{Analysis model of dual-dipole array}

In order to construct a model that enables us to change the beamwidth of the radiation pattern, we arrange two dipoles in parallel on the Tx side as one element as shown in Fig. 1 (a). The new element is named as dual-dipole element, which can adjust the HPBW of the element radiation pattern by changing the internal distance between two dipoles in one element, denoted as $\Delta d$. In addition, the transmission power of each dual-dipole element is constrained as the same as that for a conventional dipole element. To explain the definition of the HPBW, the far-field radiation pattern is utilized in this paper. Through the 3-D radiation pattern as shown in Fig. 1(a), we note that, the receiver antenna is in the $y$ direction, so the $x-z$ plane pattern does not need to be considered. When we change the $\Delta d$, we find that the HPBW of the $y$ - $z$ plane pattern hardly changes. The $\Delta d$ can only control the HPBW of the $x-y$ plane pattern. Hereafter, the HPBW of the $x-y$ plane is used to judge the change in the radiation pattern.

The near-field MIMO analysis models used in this paper are arranged in two types, horizontal and vertical, as shown in Figs. 1 (b) and 1(c), respectively. Two linear arrays consisting of identical half-wavelength dipole antennas are placed parallel face-to-face as the transmitter and receiver. The 


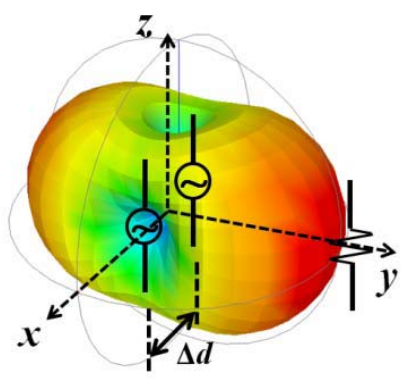

(a) Dual-dipole element

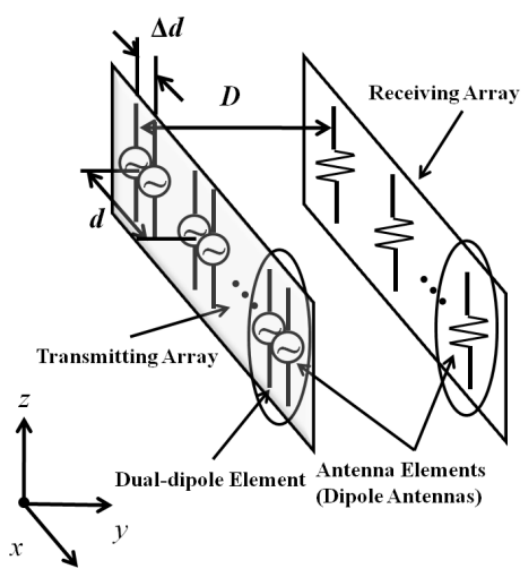

(b) Horizontal array

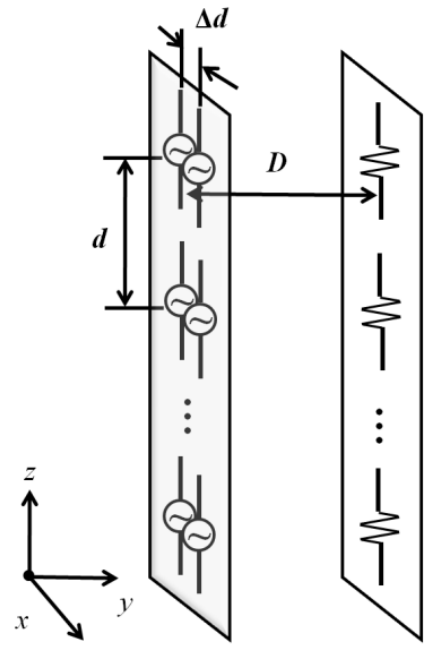

(c) Vertical array

Fig. 1. Analysis models of dual-dipole arrays in near-field MIMO.

numbers of antenna elements on Tx side $M_{T}$ and on Rx side $M_{R}$ are set to be the same, i.e., 4. The distance between two adjacent elements is denoted as element spacing $d$, the distance between the transmitter and the receiver is defined as antenna distance $D$.

\subsection{Evaluation of channel capacity}

To evaluate the performance of the near-field MIMO system, channel capacity is used as the performance index. The generalized capacity formula for the general $\left(M_{T}, M_{R}\right)$ case is given as [6]

$$
C=\log _{2} \operatorname{det}\left[I_{M_{R}}+\left(\rho / M_{T}\right) \cdot H H^{\dagger}\right] \mathrm{bps} / \mathrm{Hz}
$$

In this equation, 'det' means determinant, $I_{M_{R}}$ is the $M_{R} \times M_{R}$ identity matrix, $\rho$ is the average SNR at each receiver branch, $H$ is the normalized MIMO complex channel matrix, and ' $H^{\dagger}$ ' denotes the complex conjugate transpose of the matrix. Weight coefficients are optimized by the eigenmode beamforming algorithm.

All of the results in this paper are calculated using the Method of Moments (by EEM-MOM). 


\section{Effect of HPBW on channel capacity}

In Fig. 2 (a), we can see the relationship of the HPBW versus the channel capacity when $D$ is fixed at $1.6 \lambda_{0}$ and $d$ is fixed at $\lambda_{0}$. The straight dashed lines indicate the channel capacity when conventional dipole arrays are used on both sides in the same situation. Fig. 2 (a) indicates that the beamwidth of the radiation pattern has a great impact on the channel capacity. General speaking, when the HPBW becomes narrower, the dual-dipole MIMO system achieves a higher channel capacity than that for a conventional dipole antenna. As the HPBW changes, the channel capacity achieves a peak. The highest channel capacity is obtained when the HPBW is approximately $50^{\circ}$. The corresponding $\Delta d$ for the optimal HPBW is approximately $0.6 \lambda_{0}\left(\lambda_{0}\right.$ is the wavelength).

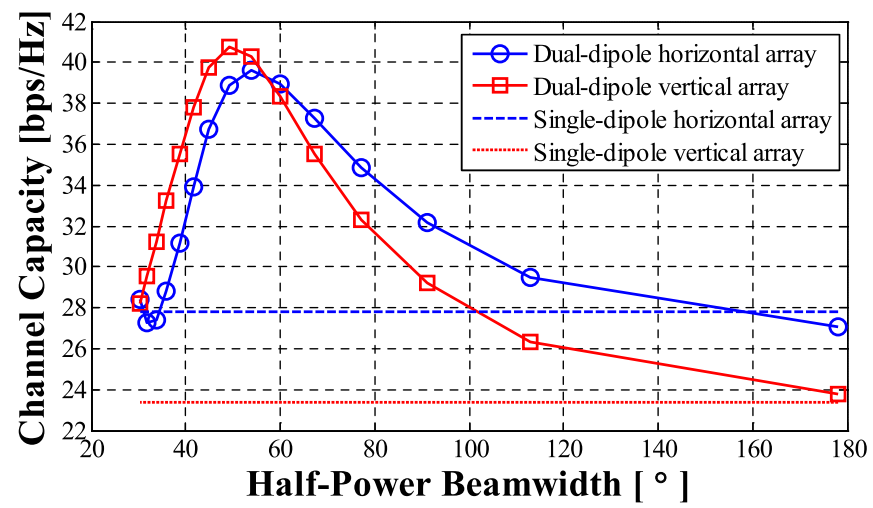

(a) Effect of HPBW on channel capacity

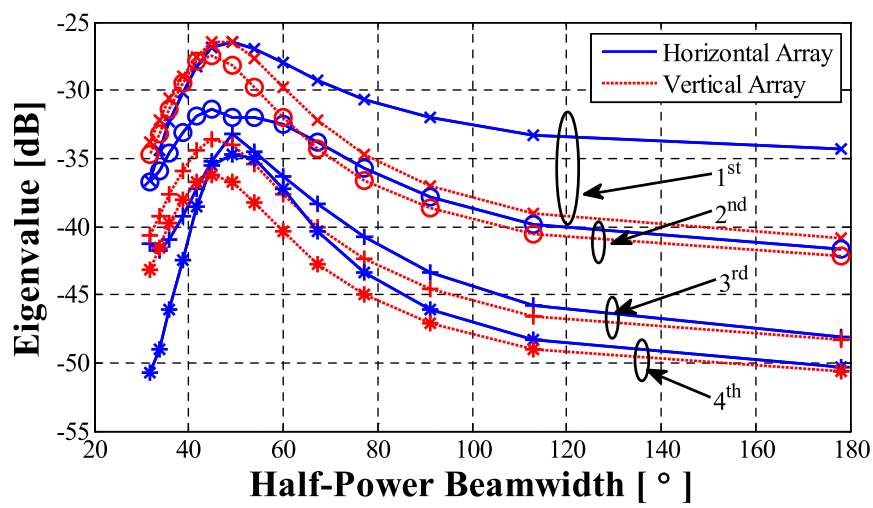

(b) Effect of HPBW on eigenvalue

Fig. 2. Effect of HPBW in near-field MIMO.

It is obvious that, when the HPBW is larger than $50^{\circ}$, the improvement in the vertical array is much considerable than that in the horizontal array. The different performances of the two types of arrangements are caused by the different spatial correlation and the different receiving SNR in each non-facing $\mathrm{Rx}$ element branch. The spatial correlation can be ignored in the vertical array but important in the horizontal array. Especially when the HPBW is near $180^{\circ}$, the spatial correlation in the dual-dipole horizontal array is larger than that of the conventional dipole array. Because the dipoles number in Tx 
increases from 4 to 8 . Therefore, the tiny increase on SNR can't cancel off the effect of the spatial correlation increment. Furthermore, in the horizontal array, as the HPBW in $x-y$ plane becomes narrower, the power received by the non-facing elements (especially the ones further from the facing one) will not increase as much as the facing one. On the other hand, in the vertical array the beamwidth in the $y$ - $z$ plane affects the SNR in each non-facing Rx element branch. The beamwidth in the $y-z$ plane will not change with the $\Delta d$, but the gain of the radiation pattern in the $y$ - $z$ plane will increase. Therefore, besides the facing element, the SNR in the other Rx branches will also increase as $\Delta d$ increases. That's why the improvement in the vertical array is larger.

The reduction of the channel capacity when HPBW is less than $50^{\circ}$ (the corresponding $\Delta d$ increases over about $0.6 \lambda_{0}$ ), is caused by the occurrence of the side lobes. The side lobes will carry off some power from the main beam, and as the HPBW becomes narrower the side lobes become larger, which leads to more spatial correlation in the horizontal array. That's why the channel capacity in the horizontal array decreases earlier than that in the vertical array. Especially in the dual-dipole horizontal array, when the HPBW is near $30^{\circ}$, the side lobes are so large that the negative impact of the spatial correlation exceeds the positive impact of SNR increment. So the channel capacity for the dual-dipole horizontal array becomes less than the results for conventional dipole hereafter. To avoid the effect of side lobes, the $\mathrm{HPBW}$ should be considered only larger than $50^{\circ}$ (the corresponding $\Delta d$ is from $0.2 \lambda_{0}$ to $\left.0.6 \lambda_{0}\right)$.

In Fig. 2 (b), the effect of the HPBW on eigenvalue distributions is plotted. When the HPBW is larger than $50^{\circ}$, we can see that in the horizontal array, although the $3^{\text {rd }}$ and $4^{\text {th }}$ eigenvalues change very obviously, the $1^{\text {st }}$ and $2^{\text {nd }}$ eigenvalues those are the principal components of channel capacity vary gently. On the other hand, all the eigenvalues in the vertical array are affected by the HPBW seriously. Hence, we can find in Fig. 2 (a) that the improvement of channel capacity in the vertical array is much more significant than that in the horizontal array. When the HPBW is larger than $50^{\circ}$, the trends of all 4 eigenvalues curves of vertical array are similar. But the trends of horizontal array are distinctly different. The vertical eigenvalues have an obvious steeper decreasing slope than the $2^{\text {nd }}$ horizontal eigenvalue, but a little gentler slope than the $1^{\text {st }}$ and $4^{\text {th }}$ horizontal eigenvalues and a similar slope as the $3^{\text {rd }}$ one. The differences among every horizontal eigenvalue will cancel out the impact of each other, and finally it shows the similar trend of channel capacity slope as that of the vertical one in Fig. 2 (a). Therefore, the improvement of channel capacity can be explained well by the variation of eigenvalues.

\section{Generality of optimum HPBW}

The effect of antenna distance $D$ and element spacing $d$ on the channel capacity in the near-field MIMO systems was clarified in [2]. The channel capacity 
will simply decrease with $D$ enlarges. However, an optimum element spacing $d_{\text {opt }}$ could be obtained at each certain $D$, because that the variation of $d$ will affect not only the SNR in each Rx element branch but also the spatial correlation. To verify the generality of the optimum HPBW mentioned in Sec. 3, we keep $D$ at $1.6 \lambda_{0}$ and change the element spacing $d$ and HPBW at the same time.

Figure 3 indicates that the $d_{\mathrm{opt}}$ still exists whatever $\Delta d$ changes, and the $d_{\mathrm{opt}}$ is $0.7 \lambda_{0}$ as the same as mentioned in [2]. However, the effect of $d$ is much less than HPBW, especially in the vertical array. In addition, the channel capacity improvement caused by adjusting HPBW is independent from $d$ changes. The optimum HPBW can be found to be approximately $50^{\circ}$.

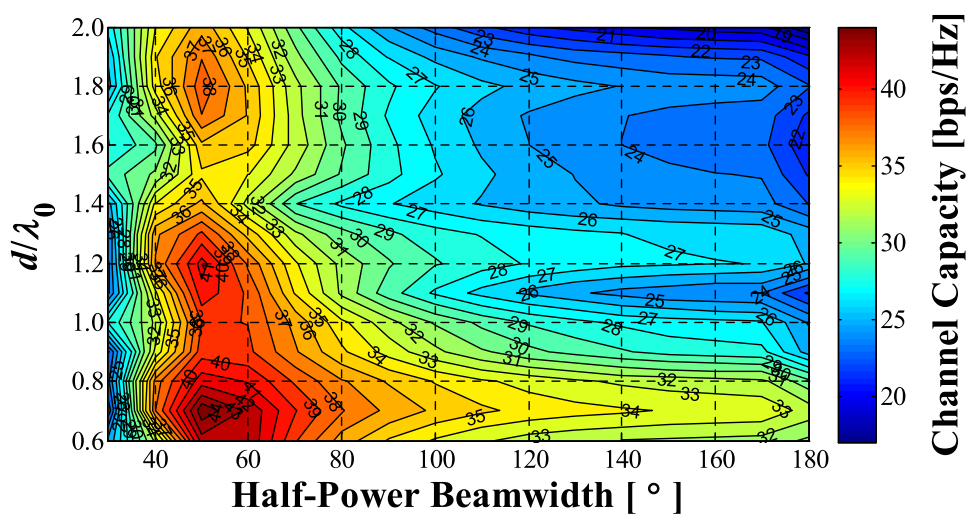

(a) Horizontal array

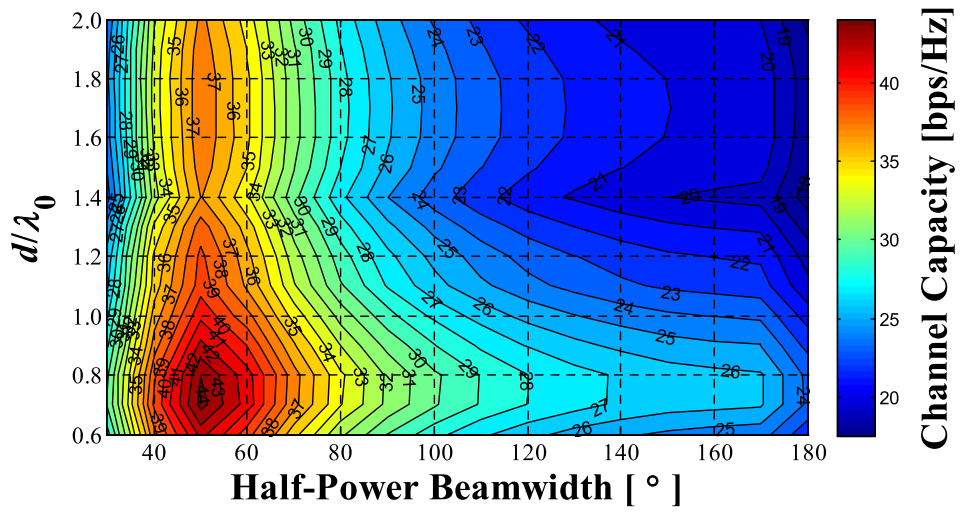

(b) Vertical array

Fig. 3. Effect of $d$ and HPBW on channel capacity when $D$ is $1.6 \lambda_{0}$.

\section{Conclusion}

This paper investigated the channel capacity in near-field MIMO systems, by clarifying the effect of antenna element radiation patterns. Horizontal and vertical dual-dipole arrays were introduced to the near-field MIMO system in this paper, and the HPBW was adjusted by changing the internal distance between two dipoles in one element. To avoid the influence of the side lobes, 
the range of the HPBW in a dual-dipole element was considered only from $180^{\circ}$ to $50^{\circ}$. The HPBW affected the system channel capacity significantly, as the HPBW decreased the channel capacity increased. And the optimum HPBW was found to be approximately $50^{\circ}$, at this time, the channel capacity considerably improved from the conventional dipole array. In addition, the effect of the HPBW is independent from the change of other situations. 